\title{
Advance measures and challenges of wound healing.
}

\section{Mukty Sinha*}

Department of Medical Devices, National Institute of Pharmaceutical Education and Research, Ahmedabad, Palaj, Gandhinagar-382355, Gujarat, India

\begin{abstract}
Skin is a protective barrier of our body and any hindrance in its continuity is a major health issue. There are many therapies available in market which acts mainly as protective to wounded area. But wound healing has many challenges like high volume of exudates, microbial infection, less perfusion. After continuous research many features have been added to the dressings. Now, the marketed dressings are highly absorbent and/or loaded with antimicrobials. Some smart dressings are also invented which have smart sensing property to show microbial load at wound site. Additional advancement in the therapy has applied vacuum at the site to remove excess exudates and provide moist and clean environment for healing. But, the advance therapies still need to be clinically proven for extensive applications.
\end{abstract}

Keywords: Wound, Exudates, VAC therapy, Absorbent dressings, Wound dressings.

Accepted on October 31, 2017

\section{Wound Management}

Skin is the biggest organ of human body and any breach in the continuity in form of wound or cut, leads to compromised health and immunity. Therefore, it is utmost necessary to treat the wound on urgent basis. The wounds can be classified as acute or chronic depending on duration of the healing. Generally, wound of more three weeks are considered as chronic wound. Since ages, the major goal of the wound dressing is to protect and cover the wound from external infections. There are a number of wound healing therapies available in the market. Apart from protective actions there are many challenges in wound healing namely, exudates management, bacterial control, fast healing. Therefore, various other functionalities or applications are added to the dressings and ultimately the role of the dressings has been shifted from protective action of the dressings to wound environment modifier.

\section{Challenges}

Despite of technological advancement the wound exudate management is still a clinical challenge. Thick or viscous exudates worsen the problem where most of the novel dressings do not possess the capabilities to manage the viscosity with exudate absorption [1]. Maceration to the periwound area is additional challenge, irrespective of availability of topical barrier application products and the innovative dressings to lock exudates. Above this, non-availability of sufficient clinical report of the assessment and documentation of wound exudates worsen the problems for the selection and cost-effective dressings. Control and removal of scar formed after wound healing is also an emerging area of research. The scar is formed due to collagen formation and it is an outcome of natural healing. The nature of scar depends on the inflammatory phase of the healing. The prolonged inflammatory phase results into excessive scar formation; whereas too less inflammation is not supporting the wound healing [2].

\section{Advancement}

Over the last few years wound management has observed significant progress. The reason is better understanding of wound healing, technological advancement and excessive clinical and scientific research. Currently a plethora of absorbent dressings on wound care are available in market which claims to have the ability to manage exudates whilst encouraging healing. However, it is becoming clear, from analysing randomized controlled trials, that some of these absorbent dressings are not meeting their expectations when applied in a clinical setting.

The clinical need has shifted towards dressing ability to encourage the healing apart from absorbing the exudates (Figure 1). The absorbent first-line dressings are made up of alginate, hydrofibre, foam, hydrocolloid and polysaccharide bead dressings. The modern dressings are equipped with other activities like modifying the proteases activity, adding growth factors, antimicrobials, or by applying smart dressings with inbuilt bio sensory mechanisms. Up to now, growth factors are normally applied topically by the clinician. The US-FDA has approved a topically applied formulation for growth factor, Platelet Derived Growth Factor isoform-BB (PDGF-BB), Regranex ${ }^{\mathrm{TM}}$, Ethicon, Livingston, $\mathrm{UK}$ to promote the chemotactic recruitment and proliferation of cells in wound repair [3]. 


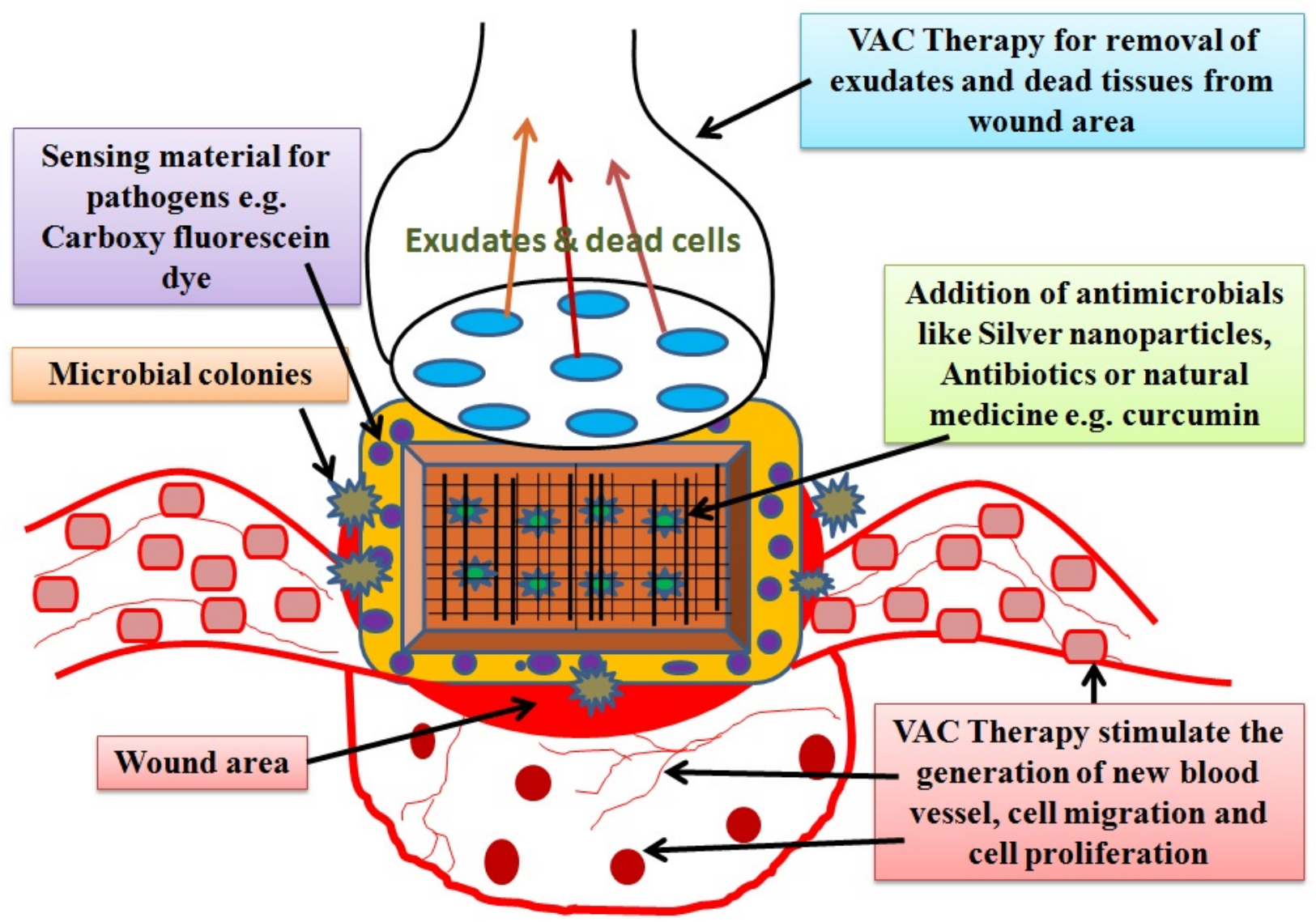

Figure 1: Schematic representation of advancements in wound management.

Silver dressings are most popular as antimicrobial dressings [4]. It is now in demand to investigate and research new and effective antimicrobial agents for infected wounds to reduce infection, subsequently reduce the level of wound exudates [5]. Chitosan and honey are under intensive research under the category. Chitosan has shown good compliance with tissues and fastened the healing [6]. Many chitosan based dressings are marketed. Honey is applied in pourable form or via a honey-saturated substrate. Despite of natural origin, it is cumbersome to apply, the viscosity of honey increases with rise temperature, cannot be applied to large wounds of diabetic patients and 'drawing' sensation has observed in some patients [7].

The next generation sensory smart wound dressings are developed with the ability to detect and report pathogenic bacteria colonization. The dressings are also capable of sensing the wound infection (Figure 1). The infection is one of the reasons for biofilm formation which eventually stimulate inflammation, increases vascular permeability and at last production of wound exudate and ultimately makes wound difficult to treat [8]. The smart dressing is made up of stabilised lipid vesicles containing a quenched carboxyfluorescein dye. The lipid vesicles are sensitive to bacterial toxins produced by pathogenic microorganisms Pseudomonas aeruginosa and Staphylococcus aureus. After coming in contact with the toxins the vesicle breaks, subsequently releases the dye and indicates pathogenic colonisation. The lipid vesicles could also be attached with a suitable antimicrobial agent and the release of the agent can be controlled by the vesicle sensitivity [9]. Molecular imprinted polymer can also be designed and applied for the interaction with the specific chemicals produced by $P$. aeruginosa. The biofilm can also be quantified using the same technique. The unique process of sensing is specifically termed as 'quorum sensing' [10].

Recent advancements of wound management have added negative pressure wound therapy (NPWT) or vacuum added closure (VAC) therapy. It has supported the clinicians to treat acute and chronic wounds and are in demand to manage complex and chronic wounds [11]. The therapy provides closed, moist- environment, facilitates wound closure by promoting granulation and perfusion, decreases edema, and removes infectious materials [12]. There are numerous other benefits of NPWT, like reduction of wound volume and suitable for healing in all type of wounds [11,12]. The technique is very useful during the preparation of the wound bed for skin grafting. However, it is difficult to remove thick exudate through the foam and dressing removal is painful in the therapy. Additionally, instillation of solution over wound bed is not possible. Therefore, next-generation pressure therapy devices have been developed to add the feature for fluid instillation (NPWT and instillation, NPWTi) in tandem for the wounds where automated delivery of solutions is advantageous. NPWTi has shown positive results in wounds at compromised places $[13,14]$. It has also proved to be effective 
in painful wounds and preferred in acutely debrided wounds and post debrided exposed bone with critical bacterial colonization [15-17].

\section{Concluding Remarks}

Effective management of exudates, infections and complex tissue growth in a variety of wounds is still a major challenge for the researchers and wound dressing manufacturers. Modern dressings have added many features to the dressings due to better understanding of the physiological characteristics of wounds and advancement in material research. Next generation wound dressings are utilizing new techniques like vacuum, instillation to tackle chronic issues at the cellular level and treat underlying causes. But the concepts are new and still need to be proven clinically. However, ongoing exhaustive research in these areas is going address all the related issues, with superior properties, and wider acceptability.

\section{References}

1. Tickle J. Effective management of exudate with AQUACEL extra. $\mathrm{Br} \mathrm{J}$ Community Nurs. 2012;17:Suppl:S38-S46.

2. Visscher MO, Bailey JK, Hom DB. Scar treatment variations by skin type. Facial Plast Surg Clin North Am. 2014;22(3):453-462.

3. Fonder MA, Lazarus GS, Cowan DA, et al. Treating the chronic wound: a practical approach to the care of nonhealing wounds and wound care dressings. J Am Acad Dermatol. 2008;58(2):185-206.

4. Cencetti C, Bellini D, Pavesio A, et al. Preparation and characterization of antimicrobial wound dressings based on silver, gellan, PVA and borax. Carbohydr Polym. 2012;90(3):1362-1370.

5. White R. Managing exudate. Nursing Times 2001;97:11-13.

6. Sinha M, Banik RM, Maiti P, et al. Incorporation and controlled release of ciprofloxacin hydrochloride using chitosan/polyethylene glycol blend polymeric scaffold, J Porous Materials 2012;20(4):799-807.

7. Lusby PE, Coombes A, Wilkinson JM. Honey: a potent agent for wound healing? J Wound Ostomy Continence Nurs. 2002; 29(6):273-274.

8. Philips PL, Wolcott RD, Fletcher J, et al. Biofilms made easy. Wounds International. 2010;1(3): 1-6.

9. Zhou J, Tun TN, Hong SH, et al. Development of a prototype wound dressing technology which can detect and report colonization by pathogenic bacteria. Biosens Bioelectron. 2011;30(1):67-72.

10. Piletska EV, Stavroulakist G, Larcombet LD, et al. Passive control of Quorum Sensing: Prevention of Pseudomonas aeruginosa Biofilm Formation by Imprinted Polymers. Biomacromolecules. 2011;12(4):1067-1071.

11. Baharestani $\mathrm{M}$, de Leon J, Mendez-Eastman S, et al. Consensus Statement: A practical guide for managing pressure ulcers with negative pressure wound therapy utilizing vacuum-assisted closure- understanding the treatment algorithm. Adv Skin Wound Care. 2008;21:1-20.

12. Blume PA, Key JJ, Thakor P. et al. Retrospective evaluation of clinical outcomes in subjects with split-thickness skin graft: Comparing V.A.C. ${ }^{\circledR}$ therapy and conventional therapy in foot and ankle reconstructive surgeries. Int Wound J. 2010;7(6):480-487.

13. Raad W, Lantis JC, Tyrie L, et al. Vacuum-assisted closure instill as a method of sterilizing massive venous stasis wounds prior to split thickness skin graft placement. Int Wound J. 2010;7(2):81-85.

14. Schintler MV, Prandl EC, Kreuzwirt G. et al, The impact of V.A.C. Instill in severe soft tissue infections and necrotizing fasciitis. Infection. 2009;37:Suppl:S1-6.

15. Gabriel A, Shores J, Heinrich C, et al. Negative pressure wound therapy with instillation: A pilot study describing a new method for treating infected wounds. Int Wound J. 2008;5(3):399-413.

16. Bernstein BH, Tam H. Combination of subatmospheric pressure dressing and gravity feed antibiotic instillation in the treatment of post-surgical diabetic foot wounds: A case series. Wounds 2005;17(2):37-48.

17. Wolvos T. The use of negative pressure wound therapy with an automated, volumetric fluid administration: An advancement in wound care. Wounds. 2013;25(3):75-83.

\section{*Correspondence to:}

Mukty Sinha

Department of Medical Devices

National Institute of Pharmaceutical Education and Research, Ahmedabad

Palaj, Gandhinagar-382355,

Gujarat, India

E-mail: mukty@niperahm.ac.in 